\title{
Effect of Guanxin V in animal model of acute myocardial infarction
}

\author{
Xiaoxiao Zhang ${ }^{1,2}$, Changle Shao ${ }^{3}$, Songyi Cheng ${ }^{2}$, Yao Zhu' ${ }^{1}$, Bo Liang ${ }^{1}$ (D) and Ning Gu ${ }^{2 *}$ (D)
}

\begin{abstract}
Background: Acute myocardial infarction (AMI) is the most serious and lethal manifestation of coronary heart disease worldwide, presenting extremely high disability and mortality. Our previous studies have shown that Guanxin V (GXV) could significantly improve the cardiac function and the blood flow dynamics, and reduce serum levels of inflammatory factors in AMI rats, thus triggering ventricular remodeling (VR) at post-AMI.

Methods: An in vivo AMl model was established in Syrian hamsters by performing the ligation of the left anterior descending coronary artery. Syrian hamsters were randomly divided into four groups, namely Sham operation group ( $n=12)$, AMl group $(n=12)$, GXV group (GXV $6 \mathrm{~g} / \mathrm{Kg} / \mathrm{d}, n=12)$, and Tranilast group (Tra $105 \mathrm{mg} / \mathrm{Kg} / \mathrm{d}, n=12$ ). Drug intervention was conducted for consecutive 8 weeks. Relative biological indicators were measured in the 4 th and 8th week, respectively.

Results: Cardiac functions were improved, and the infarcted size and heart weight index were limited in Syrian hamsters of GXV and Tra groups compared with those in AMI group. Furthermore, GXV was able to decrease the number of mast cells and chymase level in Syrian hamsters with AMI. Administration of GXV remarkably inactivated the renin-angiotension-aldosterone system, and alleviated myocardial fibrosis and cardiomyocyte apoptosis, thus slowing down VR at post-AMI.

Conclusion: GXV slows down the process of VR at post-AMI by reducing chymase level and mast cells number, as well as inactivating the reninangiotension-aldosterone system..
\end{abstract}

Keywords: Acute myocardial infarction, Ventricular remodeling, Chymase, Mast cells, Syrian hamsters, Traditional Chinese medicine

\section{Background}

Acute myocardial infarction (AMI) is a severe and lethal coronary heart disease with extremely high disability and mortality [1]. Ventricular remodeling (VR) is first proposed by Tennant and Wiggers, which involves pathological changes of ventricular dilatation, cicatrization and structural changes of the left ventricle [2]. AMI triggers morphological changes of myocardium and myocardial fibroblasts due to oxidative stress, inflammatory

\footnotetext{
*Correspondence: guning@njucm.edu.cn; jsguning@163.com

2Department of Cardiology, Nanjing Hospital of Chinese Medicine, Affiliated to Nanjing University of Chinese Medicine, Daming Road 157\#, Nanjing 210000, Jiangsu, People's Republic of China

Full list of author information is available at the end of the article
}

response, neuroendocrine regulation and other secondary lesions, thus affecting ventricular size, structure and function. Eventually, AMI-induced pathological changes lead to the incidence of VR. The occurrence of VR may further increase the risks of heart failure and malignant arrhythmias, and even cardiogenic death. Therefore, prevention and reversal of VR are beneficial to AMI treatment [3]. Early revascularization, pharmacotherapy, and device-based therapies are currently preferred to AMI patients [4].

Guanxin V (GXV) is produced by the Nanjing Hospital of Chinese Medicine Affiliated to Nanjing University of Chinese Medicine (Nanjing, China). It is applied for the treatment of coronary heart disease, myocardial

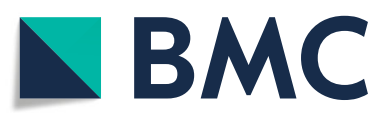

(c) The Author(s). 2021 Open Access This article is licensed under a Creative Commons Attribution 4.0 International License, which permits use, sharing, adaptation, distribution and reproduction in any medium or format, as long as you give appropriate credit to the original author(s) and the source, provide a link to the Creative Commons licence, and indicate if changes were made. The images or other third party material in this article are included in the article's Creative Commons licence, unless indicated otherwise in a credit line to the material. If material is not included in the article's Creative Commons licence and your intended use is not permitted by statutory regulation or exceeds the permitted use, you will need to obtain permission directly from the copyright holder. To view a copy of this licence, visit http://creativecommons.org/licenses/by/4.0/. The Creative Commons Public Domain Dedication waiver (http://creativecommons.org/publicdomain/zero/1.0/) applies to the data made available in this article, unless otherwise stated in a credit line to the data. 
infarction, and arrhythmia. Main components of GXV are Codonopsis (Dang Shen), Monkey Grass (Mai Men Dong), Schizandra Berry (Wu Wei Zi), Salvia Miltiorrhiza (Dan Shen), Red Peonies (Chi Shao Yao) and Rehmannia Root (Shu Di Huang) (Table 1) [5]. It is reported that Codonopsis can inhibit the apoptosis signaling AngII+Leu27-IGFII in cardiomyocytes [6]. Rehmannia Root extract can activate the SDF-1 $\alpha /$ CXCR4 cascade following AMI, thus triggering angiogenesis by reinforcing the mobilization and migration of endothelial progenitor cells [7]. Steroidal saponins is the main ingredient of Monkey Grass, which exerts various pharmacological activities, including cardiovascular protection, anti-inflammation, anti-cancer ability, antioxidation and immunomodulation [8]. Another study indicated that Tanshinone IIA inhibits myocardial apoptosis by inducing the expression of miR-133 and restraining the caspase-9 cascade [9]. Crude terpene glycoside components in Red Peonies is capable of protecting myocardial ischemia by stimulating cardiac energy metabolism and inhibiting myocardial apoptosis via activating the PI3K/AKT/mTOR signaling pathway [10]. Schizandra Berry and its main components protect against cardiovascular diseases mainly through suppressing oxidative stress, cardiomyocyte apoptosis and inflammatory response [11]. Therefore, it is believed that GXV, a traditional Chinese medicine can be applied in the treatment of heart diseases.

Tranilast (N-[3',4'-dimethoxycinnamonyl] anthranilic acid) is an anti-allergic drug that inhibits the release of chemical indicators from mast cells (MC) and basophils [12]. Besides, Tranilast is also reported to prevent against inflammatory response, cell multiplication and ventricular fibrosis [13-15]. It is reported that Tranilast prevents atrial remodeling in a canine model by inhibiting overexpression of TGF- $\beta 1$ and Rac1 [16]. In a rat model of myocardial infarction, Tranilast inhibits fibrosis and collagen production in cardiac fibroblasts, thus alleviating LV remodeling [17].

Our previous studies have shown that administration of conventional drugs combined with GXV in AMI patients significantly maintains left ventricular structure and improves function compared with those solely treated with the conventional drugs [18, 19]. Animal experimental studies also revealed that multiple preparations containing GXV obviously improve the cardiac structure and function, and suppress inflammatory response in AMI rats [20, 21]. In AMI rats, treatment of GXV combined with valsartan prolongs the survival, which slows down the increase rate of ventricular weight, alleviates pathological changes of myocardial tissues and ventricular structure, and angiotensin II type 1 receptor (AT1R) and upregulates angiotensin II type 2 receptor (AT2R) in the renin-angiotension-aldosterone system (RASS) [22]. A retrospective study demonstrated that Guanxin V is potentially safe and effective for patients with coronary artery disease, which enhances ejection fraction and output, and reveres VR [23]. The above evidences have proven that GXV is a potential natural drug used in adjusting VR at post-AMI. However, the mechanism of GXV on regulating the RAAS remains largely unknown. This study intends to observe the effect of GXV on VR and RAAS at post-AMI and the underlying mechanism.

\section{Methods}

\section{The preparation of GXV}

Main components of GXV are shown in Table 1. All herbs were recorded in the Pharmacopoeia of the People's Republic of China compiled by China Pharmacopoeia Committee. These herbs were purchased from Department of Pharmacy, Nanjing Hospital of Chinese Medicine Affiliated to Nanjing University of Chinese Medicine (Nanjing, China). The authentication of plant materials was identified morphologically by Dr. Li Wen, the chief physician of Nanjing Chinese Medicine Affiliated to Nanjing University of Chinese Medicine. The voucher specimen of this material has been deposited in Department of Cardiology, Nanjing Hospital of Chinese Medicine Affiliated to Nanjing University of Chinese Medicine. Salvia Miltiorrhiza and Schizandra Berry were extracted by refluxing 4 times the volume of $75 \%$ ethanol twice, with $2 \mathrm{~h}$ each time. Subsequently, they were mixed with the remaining drugs, and decocted in 10 times the volume of water for 3 times, with $2 \mathrm{~h}$ each time, which was finally concentrated to $1000 \mathrm{~mL}$. The preparation procedures were entrusted to the Department of Pharmacy, Nanjing Hospital of

Table 1 Introduction to the composition of GXV

\begin{tabular}{|c|c|c|c|c|}
\hline Common name of the plant & Scientific name of the plant & Latin name of the plant & Plant part & Quantity(g) \\
\hline Dang Shen & Codonopsis & Codonopsis pilosula (Franch.) Nannf. & Root & 200 \\
\hline Mondo Grass, Mai Men Dong & Monkey Grass & Ophiopogon japonicus (Linn. f.) Ker-Gawl. & Tuberous root & 100 \\
\hline Wu Wei Zi & Schizandra Berry & Schisandra Chinensis (Turcz.) Baill & Fruit & 50 \\
\hline Dan Shen & Salvia Miltiorrhiza & Salviae Miltiorrhizae Radix et Rhizoma & Root and rhizome & 200 \\
\hline Chi Shao Yao & Red Peonies & Paeoniae Radix Rubra & Root & 200 \\
\hline Shu Di Huang & Rehmannia Root & Rehmannia glutinosa (Gaert.) Libosch. ex Fisch. et Mey & Tuberous root & 200 \\
\hline
\end{tabular}


Chinese Medicine Affiliated to Nanjing University of Chinese Medicine.

\section{Reagents}

The enzyme-linked immunosorbent assay (ELISA) kits for renin, angiotensin converting enzyme (ACE), chymase, angiotensin I (Ang I) and angiotensin II (Ang II) were obtained from Meimian (Wuhan, China). The primary antibodies against $\mathrm{ACE}$, Chymase and angiotensin II type1 receptor (AT1R) were purchased from Abcam (Cambridge, UK), while those against Collagen I and Collagen III were provided by Bioss (Wuhan, China). Triphenyl tetrazolium chloride (TTC) staining kit was obtained from Sigma (Aldrich, St. Louis, USA). H\&E and Masson staining kits were provided by Solarbio (Beijing, China). In situ apoptosis detection kit was obtained from Roche (New Jersey, USA). Goat anti-rabbit antibodies and $D A B$ detection kit were purchased from ZSGB-BIO (Beijing, China). Toluidine blue (TB) staining kit was brought from LEAGENE (Huaibei, China). Acetonitrile and formic acid for ultra-performance liquid chromatography (UPLC) analysis were provided by Sigma. Tranilast capsules were brought from Pharmaceutical Company of China Pharmaceutical University (Nanjing, China).

\section{UPLC}

UPLC was performed to analyze the main ingredients of GXV and medicated sera using the UPLC-Q-TOF/ HRMS $^{\mathrm{E}}$ system (Waters, Milford, MA, USA). An ACQUITY UPLC BEH C18 $(2.1 \mathrm{~mm} \times 100 \mathrm{~mm}, 1.7 \mu \mathrm{m}$ Waters, Milford, USA) column was used with a mobile phase including A $(0.1 \%$ formic acid, $v / v)$ and B $(0.1 \%$ formic acid acetonitrile). The gradient elution was applied as follows: $98-40 \% \mathrm{~A}$ and $2-60 \% \mathrm{~B}$ for $0-14 \mathrm{~min}$; $40-2 \% \mathrm{~A}$ and $60-98 \% \mathrm{~B}$ for $14-16.5 \mathrm{~min} ; 2 \% \mathrm{~A}$ and $98 \%$ B for $16.5-18 \mathrm{~min}$; $98 \% \mathrm{~A}$ and $2 \%$ B for $18-20$ min (flow rate: $0.3 \mathrm{ml} / \mathrm{min}$, column temperature: $40^{\circ} \mathrm{C}$ ).

The high-resolution ion mobility liquid mass spectrometer SNAPT G2-Si Q-TOF/HRMS was used for mass spectrometry analysis in the mode of positive and negative ions using the ESI source. The high-purity $\mathrm{N}_{2}$ was utilized as the auxiliary spray ionization and dissolvent gas. The following parameters were applied: Flow rate of drying gas: $10 \mathrm{~mL} \cdot \mathrm{min}^{-1} ; \mathrm{N}_{2}$ temperature: $120^{\circ} \mathrm{C}$; Atomization gas pressure: $310 \mathrm{kPa}$; Flow rate of dissolvent gas: $900 \mathrm{~L} \cdot \mathrm{h}^{-1}$; Flow rate of cone blowback: $50 \mathrm{~L} \cdot \mathrm{h}^{-1}$; Capillary ionization voltage: $500 \mathrm{~V}$; Cone voltage: $40 \mathrm{~V}$; Collision energy: $40-65 \mathrm{eV}$ and scanning range of quadrupole: $50-1200 \mathrm{Da}$.

\section{Experimental animals}

Animal procedures were approved by the Animal Ethics Committee, Nanjing University of Chinese Medicine
(Nanjing, China). Sixty male Syrian hamsters $(110 \pm 20 \mathrm{~g}$, 8 weeks old) were provided the Vital River Laboratory Animal Technology Co. LTD (Beijing, China). They were raised in the Laboratory Animal Centre, Nanjing University of Chinese Medicine (12 h light/dark cycle, temperature: $23 \pm 1{ }^{\circ} \mathrm{C}$, humidity: $55-60 \%$ ). Syrian hamsters were given to free accesses to the food and water.

An in vivo AMI model was established in Syrian hamsters by performing the ligation of the left anterior descending coronary artery (LAD) [24, 25]. The hamsters were anesthetized by subcutaneous injection of $2 \%$ pentobarbital sodium $(4 \mathrm{ml} / \mathrm{kg}$, Sigma). The electrocardiography (Lead ii, ECG-9620P, Shanghai optoelectronic medical instrument Co., LTD, Shanghai, China) was performed by connecting electrodes to subcutaneous extremities of hamsters. After shaving, iodophor disinfection and cutting off the neck skin, hamster trachea was exposed for the following blunt dissection of muscles. Mechanical ventilation was conducted by endotracheal intubation though a ventilator (ALC-V8S, Shanghai Alcott biotechnology Co., LTD, Shanghai, China). Ventilation indexes were set as follows: Tidal volume: 4-5 ml; The ratio of inhalation to respiration 1 : 2; Respiration rate: 75 times $/ \mathrm{min}$. The heart was exposed by cutting off the skin alongside the 3rd to 4th intercostal level of the left chest. Subcutaneous tissues and muscles were bluntly separated layer by layer. After exposure of the heart, LAD was performed by suturing at $2 \mathrm{~mm}$ below the lower margin of the left auricle using the $7-0$ silk suture. Decreased myocardial motion, pale myocardium and $0.2 \mathrm{mV}$ elevation of ST segment indicated the successful establishment of AMI model in Syrian hamsters. Finally, the exposed chest was sutured layer by layer and the residual gas was squeezed out. Extubation was performed until the heart rhythm was stable. Syrian hamsters in the Sham operation group received the same procedures except for ligation. After animal procedures, $4 \mathrm{U}$ penicillin (Zhongnuo pharmaceutical Co. LTD, Shijiazhuang, China) was subcutaneously administrated for consecutive 3 days to prevent postoperative infection.

\section{Experimental design}

A total of $48 / 60$ (80\%) hamsters survived after procedures. According to the postoperative survival, the hamsters were randomly divided into four groups, namely Sham operation group $(n=12)$, AMI group $(n=12)$, GXV group (GXV $6 \mathrm{~g} / \mathrm{Kg} / \mathrm{d}, n=12)$ and Tranilast group (Tra $105 \mathrm{mg} / \mathrm{Kg} / \mathrm{d}, n=12$ ). Hamsters in AMI group, GXV group and Tra group were operated by LAD to establish the AMI. The equivalent doses of GXV and Tranilast administrated in hamsters were converted according to the adult doses of a standard weight. The same volume of normal saline was administrated in 
those of Sham operation group and AMI group. Intragastric administration was given on the 3rd day postoperatively and once a day for 8 weeks, which was independently performed by the investigator Xiao-Xiao Zhang based on the randomization table who was the only one being aware of the treatment group allocation. Cardiac markers were measured in the 4th and 8th week, respectively. The hamsters were anesthetized by subcutaneous injection of $2 \%$ pentobarbital sodium (4 $\mathrm{ml} / \mathrm{kg}$ ) for collecting blood collection from abdominal aorta, and their hearts. All hamsters were sacrificed by cervical dislocation and their carcasses were concentrated destructed by the Laboratory Animal Centre, Nanjing University of Chinese Medicine.

\section{Determination of cardiac functions}

The hamster was anesthetized and fixed at the supine position. M-mode echocardiograph was obtained using a small-animal ultrasound probe (VisualSonics Vevo2100, Toronto, Canada) on the long axis of the parastolic left ventricle. The following indicators were recorded: Left ventricular end-diastolic inner diameter (LVIDd), left ventricular end-systolic inner diameter (LVIDs), left ventricular end diastolic volume (LVEDV), left ventricular end systolic volume (LVESV), ejection fraction (EF), fractional shortening (FS), left ventricular posterior wall end-diastolic thickness (LVPWd) and left ventricular posterior wall end-systolic thickness (LVPWs).

\section{Detection of heart weight index and myocardial infarction size}

The body weight of each hamster was recorded before sacrifice. After humane sacrifice, the heart was harvested and weighed. The heart weight index (HWI) was then calculated: $\mathrm{HWI}=$ heart weight $/$ body weight $(\mathrm{HW} / \mathrm{BW}$, $\mathrm{mg} / \mathrm{g}$ ). Then, the heart was frozen in $-20^{\circ} \mathrm{C}$ for $10 \mathrm{~min}$, sliced into $2 \mathrm{~mm}$ sections and stained in $2 \%$ tetrazolium chloride at $37^{\circ} \mathrm{C}$. Thirty minutes later, sections were fixed in $10 \%$ neutral formalin. Infarcted myocardium turned pale and the remaining normal one was dark red. Stained heart sections were captured and infarcted size was calculated using Image J software (NIH, Bethesda, USA).

\section{Histopathology and immunohistochemistry}

The heart was cut off from its surrounding vessels. The left ventricle of the heart, including infarcted and noninfarcted areas, was taken and fixed in $4 \%$ paraformaldehyde for $48 \mathrm{~h}$ and paraffin embedded. Sections were sliced into $3-\mu \mathrm{m}$ thickness.

Histopathological changes and cardiac fibrosis were observed and captured by a microscope (BX51T-PHDJ11, Olympus, Tokyo, Japan) following H\&E, and Masson staining.
MCs were stained by TB and counted by the microscope. Paraffin-embedded sections were dewaxed, hydrated, stained with TB working solution for 10-20 min and washed with distilled water for 3 times. Then, the slices were differentiated with $0.5 \%$ glacial acetic acid and washed twice with distilled water. At last, slices were dehydrated with $95 \%$ ethanol for 3-5 s, dehydrated with anhydrous ethanol for $1 \mathrm{~min} \times 2$ times, penetrated with xylene for $2 \mathrm{~min} \times 2$ times, and sealed with neutral gum. MCs were stained blue purple, and the nuclei were light blue.

Immunohistochemical staining was performed by the SP method. Paraffin-embedded sections were dewaxed, hydrated and subjected to antigen retrieval in citric acid buffer for $20 \mathrm{~min}$. Non-specific antigens were inactivated by incubation in $3 \%$ hydrogen peroxide for $10 \mathrm{~min}$. After blockage in normal goat serum for $20 \mathrm{~min}$, slices were incubated with corresponding primary antibodies at $4{ }^{\circ} \mathrm{C}$ overnight. The biotinylated secondary antibody (IgG) was applied as the negative control. On the next day, slices were washed in phosphate buffer saline (PBS) for 3 times, incubated with horseradish peroxidase-labeled streptomycin working solution (S-A/HRP) at $37^{\circ} \mathrm{C}$ for $20 \mathrm{~min}$, and washed in PBS for 3 times. The mixture of color reagent A, B, C (a drop of each, respectively) and $1 \mathrm{ml}$ of distilled water were prepared and applied on the sections. Sections were then thoroughly washed for 6 min, sealed by neutral resin and finally captured using a microscope.

\section{TUNEL/DAB immunofluorescence}

Paraffin-embedded left ventricle heart sections (including infarcted and non-infarcted areas) were dewaxed, dehydrated and incubated with protease $\mathrm{K}(20 \mu \mathrm{g} / \mathrm{ml}$ dissolved in Tris/ $\mathrm{HCl}, \mathrm{pH} 7.4 \sim 8.0$ ) at room temperature for $15-30 \mathrm{~min}$. After washing in PBS twice, sections were reacted with $50 \mu \mathrm{l}$ of TUNEL reaction mixed solution in a wet box at $37^{\circ} \mathrm{C}$ for $1 \mathrm{~h}$. Glass slides were covered to prevent evaporation and ensure the evenly distribution of TUNEL reaction mixture. TUNELpositive staining was then analyzed under a fluorescence microscope after 3 times washing with PBS. Subsequently, sections were incubated with $50 \mu \mathrm{l}$ of conversion agent-POD in the wet box at $37^{\circ} \mathrm{C}$ for $30 \mathrm{~min}$. Incubation of $50 \sim 100 \mu \mathrm{l}$ of DAB substrate solution at room temperature for $10 \mathrm{~min}$ was conducted following 3 times washing in PBS. At last, nuclei were counterstained by hematoxylin, sealed and captured.

\section{Western blotting (WB) analysis}

Total protein was extracted from the left ventricle heart tissues (including infarcted and non-infarcted areas) of Syrian hamsters. Protein concentration was determined by the bicinchoninic acid (BCA) protein assay kit (Beyotime, Nanjing, China). The concentration of each sample 
was adjusted to the equal one by adding the calculated loading buffer. Separated proteins were transferred onto polyvinylidene fluoride membranes (PVDF, Millipore, Billerica, USA) after the 10\% SDS-polyacrylamide gel electrophoresis. Non-specific antigens were blocked using the $5 \%$ bovine serum albumin (BSA, Beyotime, Nanjing, China) for $1 \mathrm{~h}$ at RT. Afterwards, membranes were reacted with the diluted primary antibodies (1: 1000) at $4{ }^{\circ} \mathrm{C}$ overnight, washed with TBST, and reacted with the diluted horseradish peroxidase-conjugated secondary antibodies for $1 \mathrm{~h}$ at RT. Protein bands were visualized by the enhanced chemiluminescence (ECL) detection regent (Millipore, Bedford, USA) and a chemiluminescence image analyzer (GE Healthcare, Uppsala, Sweden). Image J software was used to analyze the bands.

\section{ELISA}

Relative levels of renin, ACE, chymase, Ang I and Ang II in serum and the left ventricle heart tissues (including infarcted and non-infarcted areas) of Syrian hamsters were detected by ELISA according to the manufacturer's recommendations.

\section{Statistical analyses}

All values were expressed as mean \pm standard deviation. GraphPad Prism 8.0 (GraphPad Software Inc., San Diego, CA, USA) software was used for statistical analyses. Two-way ANOVA was used to compare the differences between groups. $P<0.05$ was considered as statistically significant.

\section{Results}

\section{UPLC profile and constituent contents of GXV}

The main components of medicated sera of GXV were analyzed by comparing with the second-order chromatogram and serum pharmacochemistry method. The total ions chromatograph (TIC) was a chromatogram generated after collecting all ions, which had a high universality. Medicated sera of GXV mainly contained 15 components, including Codonopiloside A, Lobetyolin, dihydrocatalpol, Ctlpol, RehmanniosideA, and others (Table S1).

\section{GXV effectively improved cardiac function in AMI hamsters}

EF and FS were significantly enhanced after treatment of GXV and Tranilast for 8 weeks compared with those of AMI group (EF: $61.94 \pm 3.76 \%$ in GXV group and $59.98 \pm 4.683 \%$ in Tra group v.s. $37.82 \pm 4.73 \%$ in AMI group; FS: $37.07 \pm 3.90 \%$ in GXV group and $33.03 \pm$ $9.08 \%$ in Tra group v.s. $20.50 \pm 3.68 \%$ in AMI group). LVIDd and LVIDs were obviously reduced after treatment with GXV and Tranilast in the 8th week (LVIDd:
$5.96 \pm 0.51 \mathrm{~mm}$ in GXV group and $5.92 \pm 0.24 \mathrm{~mm}$ in Tra group v.s. $7.31 \pm 0.46 \mathrm{~mm}$ in AMI group; LVIDs: $3.37 \pm 0.52 \mathrm{~mm}$ in GXV group and $3.51 \pm 0.94 \mathrm{~mm}$ in Tra group v.s. $5.03 \pm 0.43 \mathrm{~mm}$ in AMI group). LVEDV and LVESV were also obviously reduced after treatment with GXV and Tranilast in the 8th week (LVEDV: $137.32 \pm 36.01 \mu \mathrm{L}$ in GXV group and $159.36 \pm 24.51 \mu \mathrm{L}$ in Tra group v.s. $226.09 \pm 47.31 \mu \mathrm{L}$ in AMI group; LVES V: $64.29 \pm 24.07 \mu \mathrm{L}$ in GXV group and $77.71 \pm 23.67 \mu \mathrm{L}$ in Tra group v.s. $140.83 \pm 31.86 \mu \mathrm{L}$ in AMI group). No significant differences in EF, FS, LVIDd and LVIDs were found between GXV group and Tra group (Fig. 1a-i). Thus, cardiac structure and function were improved after treatment of GXV and Tranilast.

\section{GXV reduced the myocardial infarction area and HWI}

The infarcted myocardial tissue was stained to be pale, while the normal part was dark red by TTC. Infarcted size was calculated by the ratio of the infarcted area (pale) and the total area measured by Image J. Notably, infarcted size was obviously narrowed regardless of the treatment for 4 or 8 weeks $(P<0.05$ and $P<0.01$, respectively, Fig. 2a). The HWI was calculated as the ratio of the heart weight of body weight. HWI (mg/g) was significantly different between the GXV group and AMI group $(3.37 \pm 0.15$ v.s. $3.58 \pm 0.27$, Fig. $2 \mathrm{~b})$. The myocardial infarct size and HWI decreased after treatment of GXV.

\section{GXV ameliorated the pathological changes and fibrosis in myocardium at post-AMI}

To observe the function of GXV in alleviating myocardial fibrosis, $\mathrm{H} \& \mathrm{E}$, Masson and immunohistochemical staining in myocardium were performed. As shown in Fig. 3a, cardiomyocytes in hamsters of Sham operation group were oval. Nuclei were round-shaped and large, which were distributed in the center of cells. Cardiomyocytes were evenly stained and enriched in sarcoplasma. In AMI group, cardiomyocytes were disorderly arranged with significant damages. Nuclei were elongated and interstitial fibrosis was severe. Large-scale apoptosis and necrosis, as well as infiltration of inflammatory cells could be seen. In GXV and Tra group, disorderly arranged cardiomyocytes, interstitial fibrosis and inflammatory response were alleviated (Fig. 3b-g). Cardiac edema was observed. As shown in Fig. 3b, collagenous fiber was dyed green. Collagen I and III were dyed brown in Fig. 3c-d. The CVF value of collagenous fiber, and MOD value of collagen I and III decreased obviously with the treatment of GXV compared with AMI group after 8 weeks (CVF: $0.161 \pm 0.011$ v.s. $0.275 \pm 0.017$; MOD of collagen I: $0.229 \pm 0.014$ v.s. $0.313 \pm 0.005$; MOD of collagen III: $0.247 \pm 0.008$ v.s. $0.281 \pm 0.002$, 
Fig. 3e-g). The above results all demonstrated that GXV alleviated myocardial fibrosis at post-AMI.

\section{GXV inactivated the RAAS}

To explore how GXV alleviated myocardial fibrosis after AMI, potential change of the RAAS was detected (Fig. 4). As shown in Fig. 4a and Fig. 4e, the level of Renin had no difference before and after GXV treatment. In particular, relative levels of Ang I, Ang II, AT1R were significantly reduced after treatment. The level of chymase also decreased obviously with the treatment of GXV and Tra. Therefore, in the RASS, the Renin was activated after AMI, but the activation of its products (Ang I, Ang II and AT1R) and chymase was significantly reversed by GXV. It is suggested that GXV protected myocardial fibrosis after AMI by inactivating the RAAS.

\section{GXV significantly relieved AMI-induced myocardial apoptosis}

As shown in Fig. 5, there were a large number of brownstained apoptosis cells gathered together in the heart tissues from A MI hamsters, whilst the apoptosis was significantly relieved by the treatment of GXV and Tranilast.

\section{GXV could reduce the number of MCs}

After AMI, the number of MCs was apparently enhanced. Treatment of GXV and Tranilast markedly decreased the number of MCs in hamsters at post-AMI (Fig. 6).

\section{Discussion}

Myocardial infarction and heart failure are major causes of death in the world. A relevant report estimated that the number of patients with heart failure will rise to more than eight million by 2030 [26]. In recent years, the incidence of heart failure at post-AMI has gradually increased. VR plays an important role in the development of myocardial infarction [4]. In the early stage of AMI (several days following AMI), abundant inflammatory cells destroy the collagen scaffold, leading to the expansion of the infarcted myocardium. During this process, fibroblasts are accumulated in the lesioned site, leading to the formation of a new collagen matrix. As a result, the newly formed scar tissues help maintain the structure and shape of ventricles [27-29]. After a few weeks to months, increased load on the surviving cardiomyocytes leads to cardiomyocyte hypertrophy, which eventually results in left ventricular dilatation and progressive changes of mitral valve [30-33]. Once the left ventricle fails to compensate, the left ventricular ejection fraction drops. Therefore, left ventricular diameter and volume can be used as echocardiographic indexes for VR. In this experiment, LVIDd, LVIDs, LVEDV and LVESV were obviously reduced after treatment with GXV and Tranilast in the 8th week.

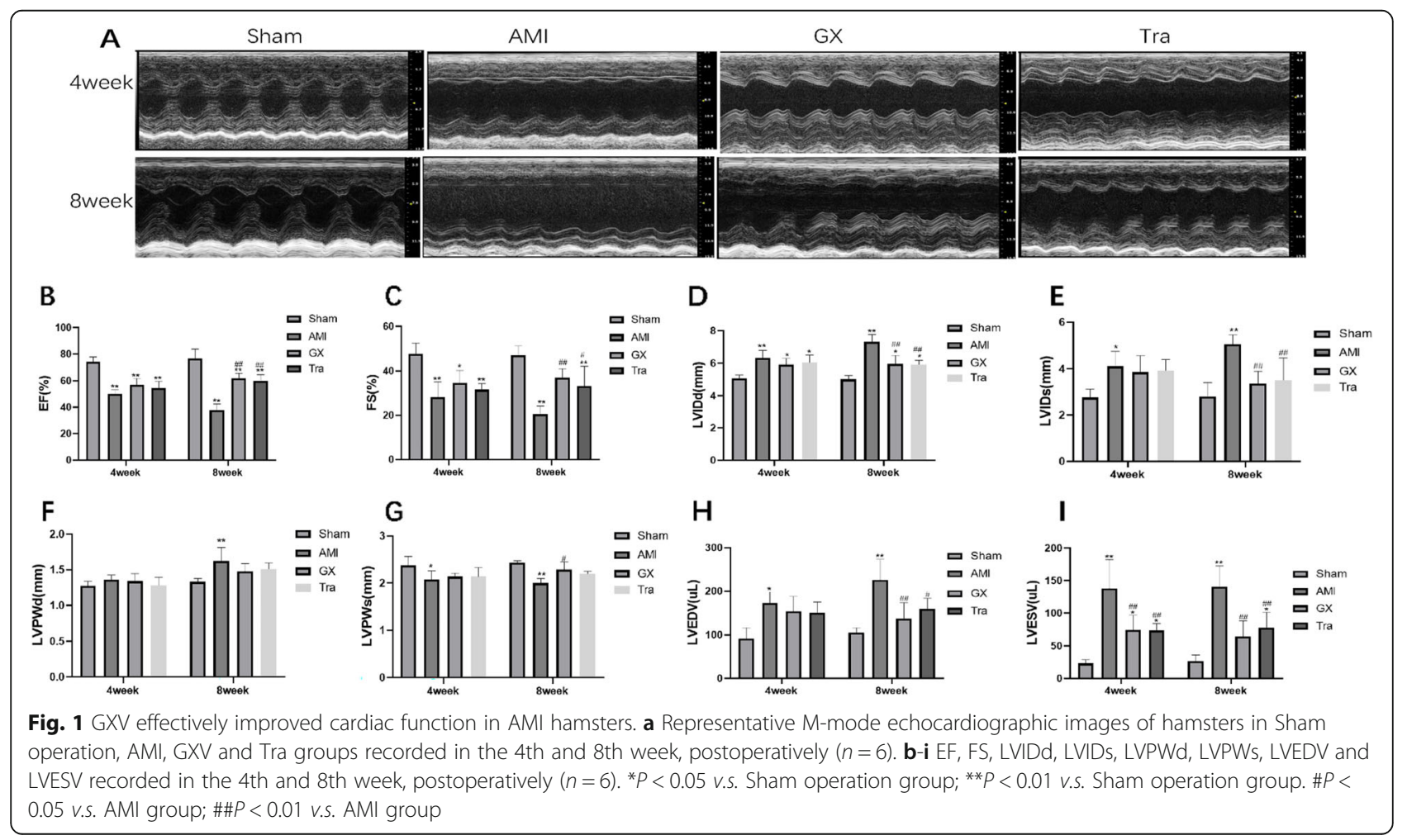



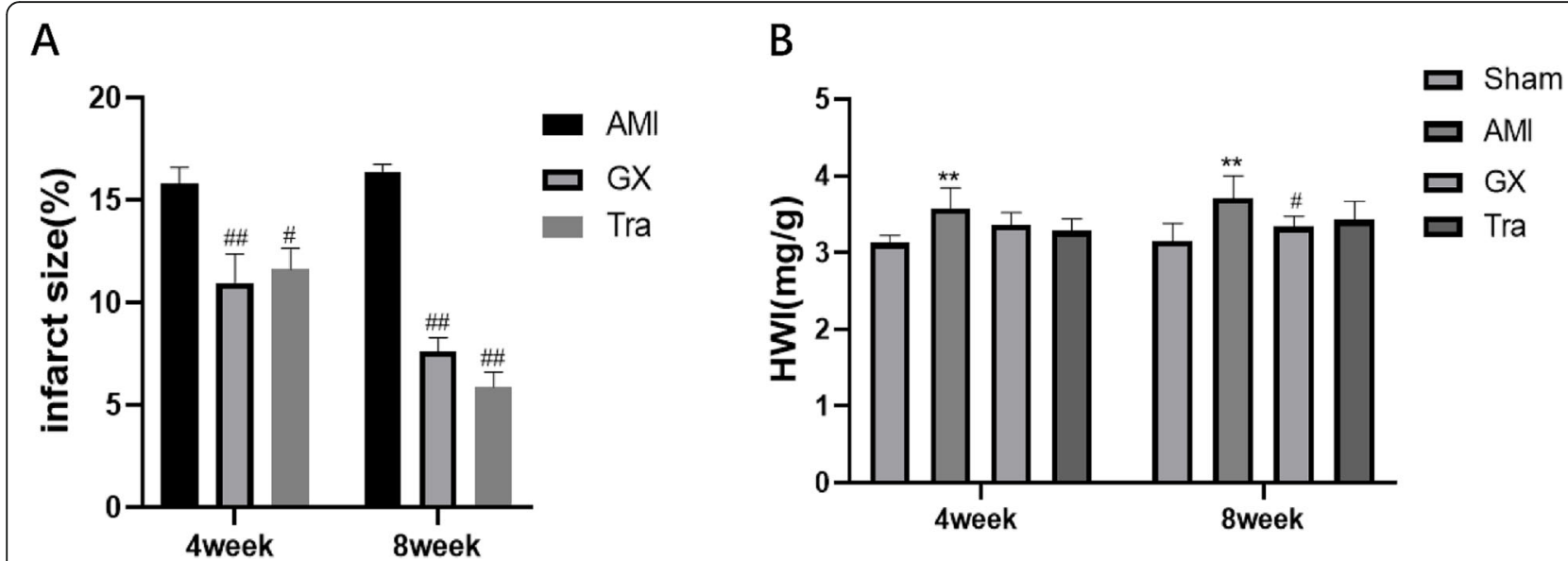

Fig. 2 Representative images of TTC staining and analysis of HWI. a The infarct size in AMI, GXV and Tra groups in the 4th and 8th week, postoperatively $(n=3)$. b HWI in AMI, GXV and Tra groups in the 4th and 8th week, postoperatively $(n=6) .{ }^{*} P<0.05$ v.s. Sham operation group; ${ }^{* *} P<0.01$ v.s. Sham operation group. $\# P<0.05$ v.s. AMl group; \#\# $<0.01$ v.s. AMl group

The activation of RAAS was excessive in the occurrence and development of VR after AMI and can lead to the perpetual vasoconstriction, LV hypertrophy, sympathetic nervous activation [34]. Ang II can either have a direct toxic effect on cardiomyocytes or bind to AT1R. Subsequently, apoptosis, cardiac hypertrophy and production of type I collagen are triggered [35]. The binding between Ang II and AT2R exerts a protective effect on the heart. Their transfection efficacies have been confirmed in some trials, such as the SAVE trial and the OPTIMMAL clinical trial [36-38]. At present, angiotensin-converting enzyme inhibitor (ACEI), angiotensin receptor blockers (ARBs) are commonly used in clinical application to prevent myocardial remodeling following myocardial infarction [39]. However, there are some limitation about these drugs in clinical. ACEI and ARBs can increase the activity of plasma Renin, which are only capable of temporarily reducing plasma

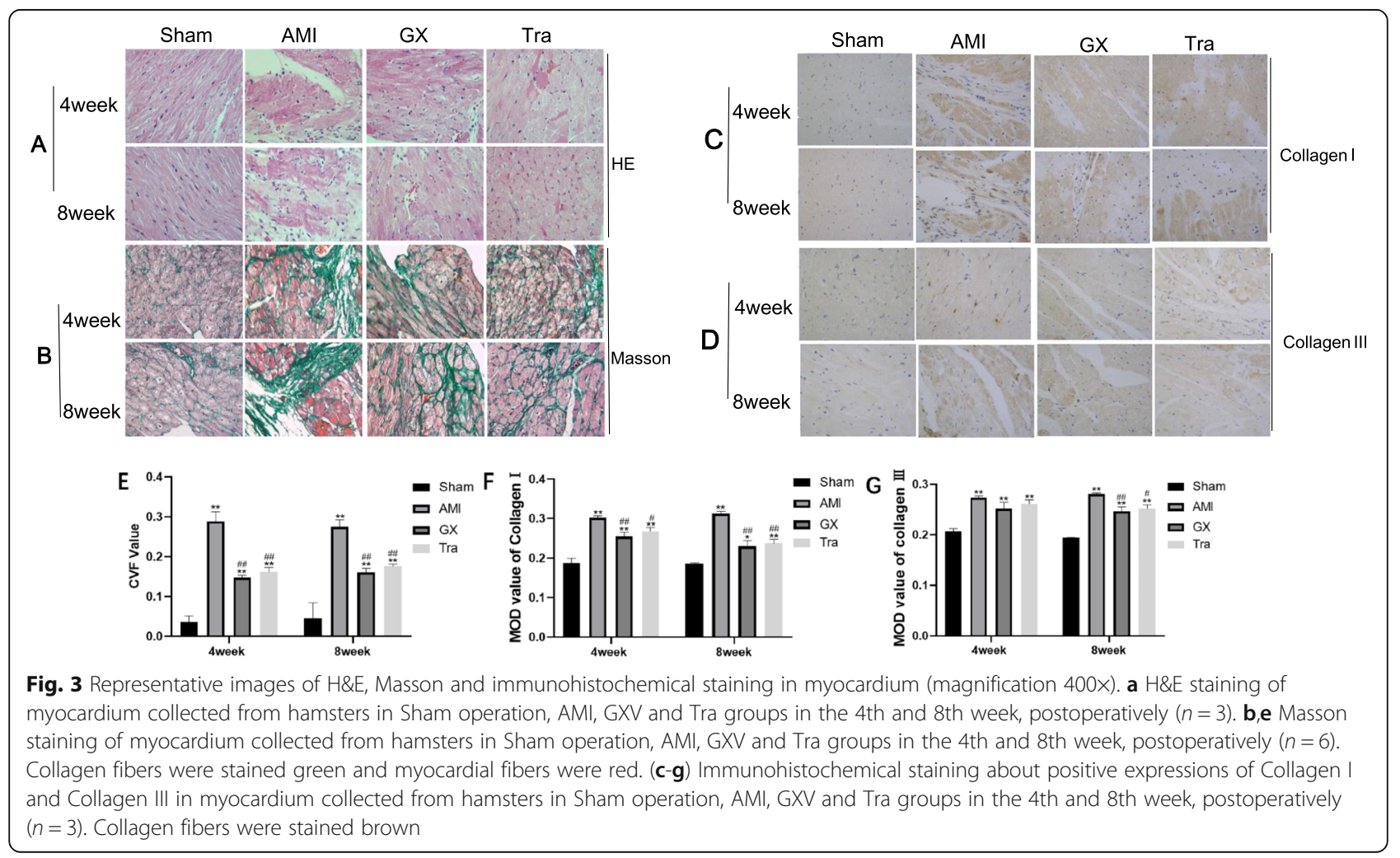




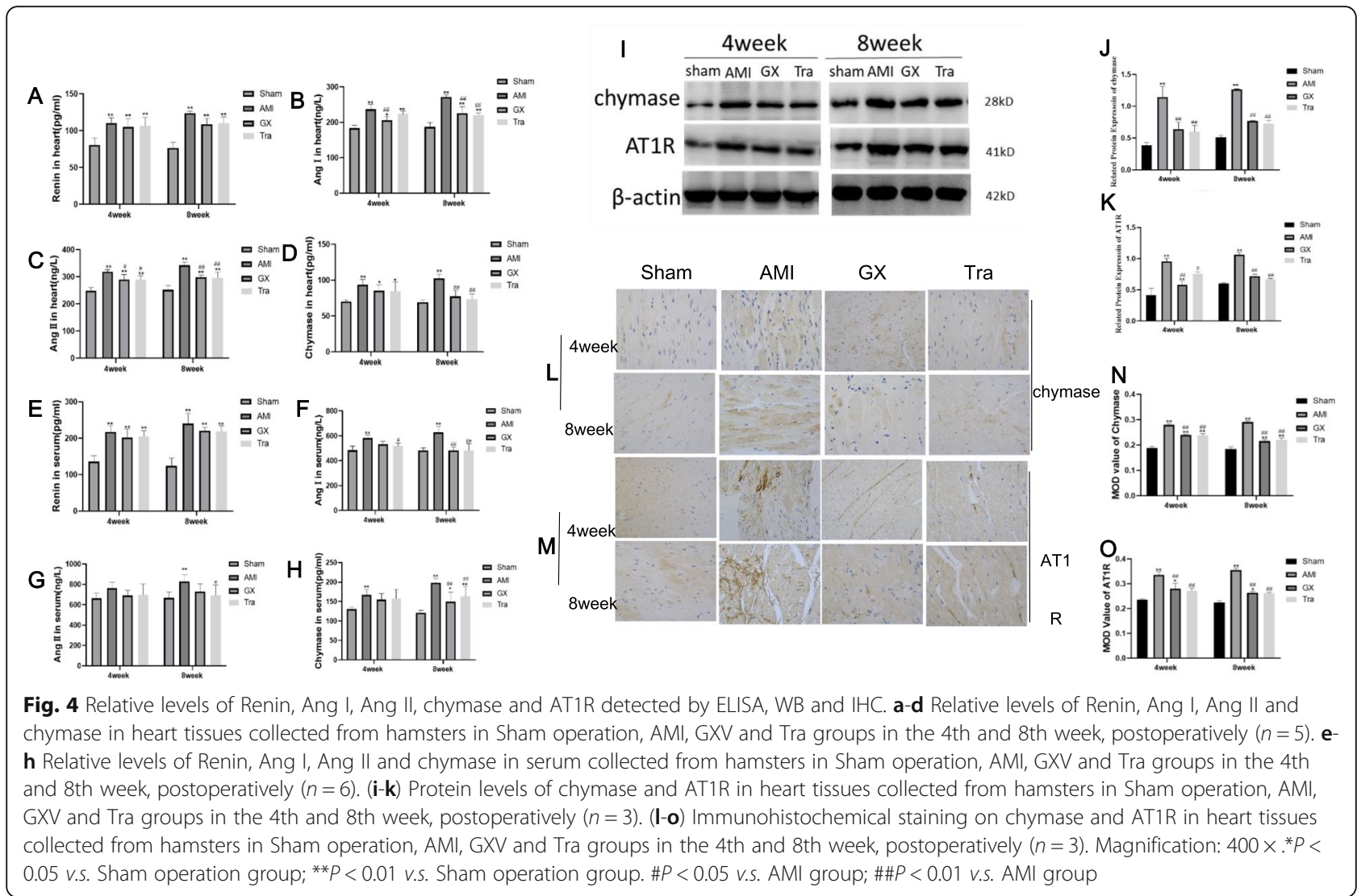

aldosterone levels. They cannot block mineralocorticoid receptors. DREAM (Diabetes Reduction Assessment with Ramipril and Rosiglitazone Medication Trial) found that the inhibitory effect of a single treatment with ACEIs on the RAAS is far more less. About $50 \%$ of patients with chronic heart failure will develop ACE escape [40]. Therefore, in addition to the classic RAAS, the non-ACE-dependent Ang II production pathway has gradually been concerned. It contains a variety of proteases (e.g. chymase, cathepsin G, Tonin, kallikrein), of which the chymase pathway is the most important.

MCs are abundant in the heart, and they are vital in many diseases, including cancer, allergenic and inflammatory diseases. Specific proteases (including tryptase, chymase, and carboxypeptidase A3) in MCs are encapsulated in granules, which are released through the liganddependent pathway during the process of inflammatory response [41]. Growing evidences have indicated that chymase in MCs is one of the pivotal factors leading to tissue fibrosis and remodeling processes via the products of vasoactive and pro-inflammatory substances [42]. Chymases, members of the serine protease family, have a wide range of peptide hydrolysis activities. They are mainly expressed in MCs, fibroblasts, vascular endothelial cells and granulocytes. When AMI occurs, the RAAS is activated and the catalytic activity of chymase is 20 times higher than that of ACE during the transformation from Ang I to Ang II [43]. Chymase distributed in MCs is non-functional, which exerts protease activity only after MCs degranulation under the stimulus of inflammatory cytokines or tissue damages [44]. Numerous studies have supported the role of MCs and chymase in VR and heart failure. Therefore, it is particularly important to develop drugs that can inhibit the activity of chymase and decrease the number of MCs, thereby preventing VR at post-AMI.

In this experiment, echocardiograph findings indicated EF decline and enlargement in the left ventricular cavity in AMI hamsters. Pathological staining results revealed disorderly arranged and damaged cardiomyocytes, largescale apoptosis and necrosis, inflammatory cell infiltration and severe interstitial fibrosis in the heart of AMI hamsters. Immediately following the onset of AMI, enhanced activity of RAAS, stimulated transformation from Ang I to Ang II and the binding of Ang II to the AT1 receptor induced the release of collagens, further aggravating cardiac fibrosis and VR. During the process, chymase and MCs exerted indispensable roles. After cardiac necrosis, the inflammatory reaction promotes the MCs to release chymase, which catalyzes the transformation of Ang I to Ang II. Notably, GXV treatment for 4 or 8 weeks significantly protected cardiac function and 

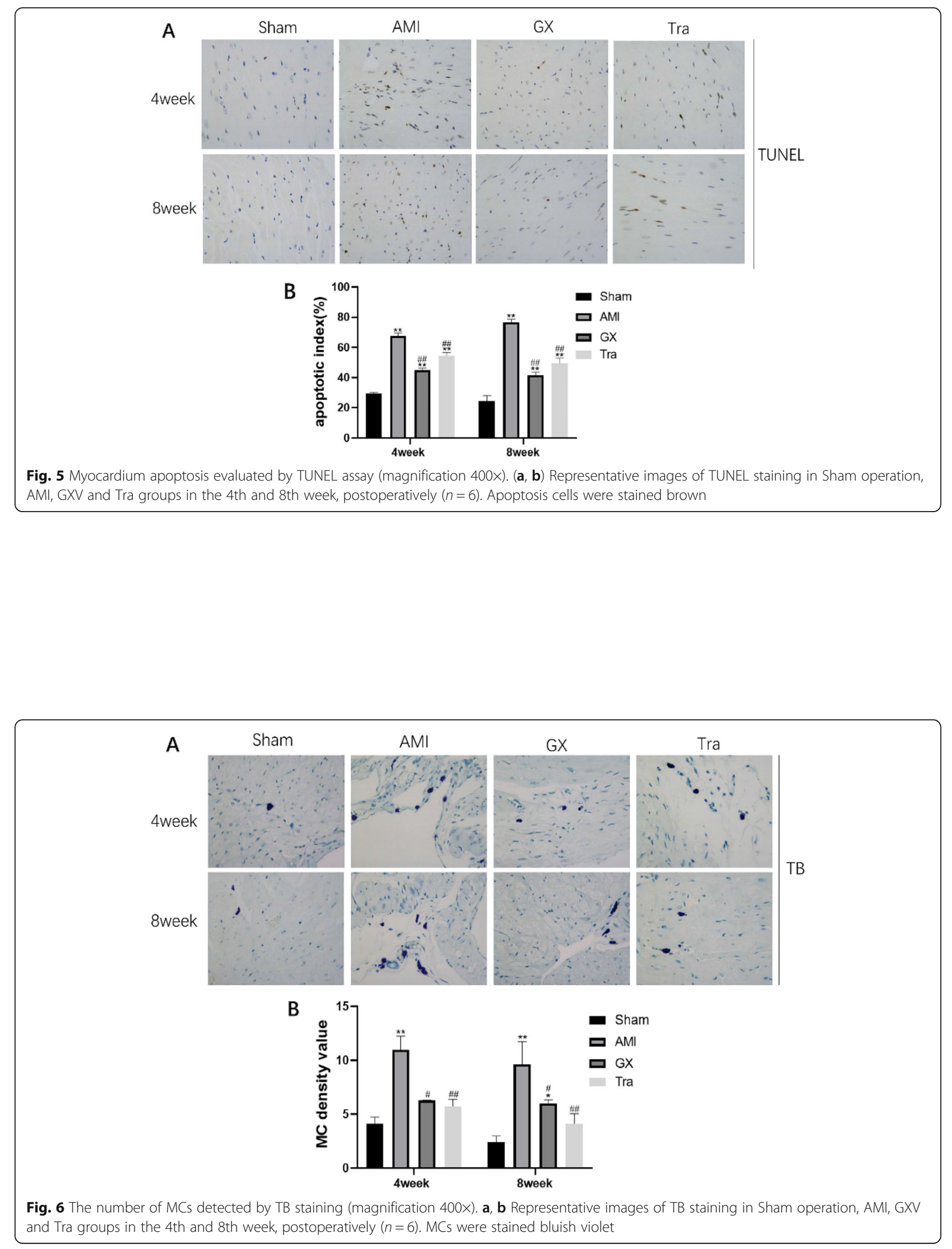
inactivated the RAAS, manifesting as decreased Ang II and collagen levels, alleviated cardiac fibrosis, reduced LV diameter (LVIDd, LVIDs) and volume (LVEDV, LVESV), increased EF and FS. MCs number and chymase level were significantly reduced after treatment with GXV. Similar results were obtained in Tra group as well. It is well known that Tranilast is a specific inhibitor of MCs, and it is effective in delaying ventricular remodeling. Therefore, it was selected as a positive control drug in our experiment. Our findings demonstrated solid evidences that Guanxin V could significantly reduce infarct size, protect cardiac function, reduce cardiac fibrosis and VR at post-AMI, which provided a novel direction in traditional Chinese medicine treatment of AMI. In addition, GXV could reduce the degree of myocardial apoptosis, which protected the cardiac function to a certain extent.

Our study clarified that GXV intervened the RAAS through the non-ACE pathway following AMI. Specific signaling pathways and targets during this process remain largely unclear, which will be further explored in our future experiments.

\section{Conclusion}

GXV protects VR at post-AMI by decreasing chymase level, MCs number and inactivating the RAAS.

\section{Supplementary Information}

The online version contains supplementary material available at https://doi. org/10.1186/s12906-021-03211-7.

Additional file 1: Table S1. Summarizes of medicated sera of Guanxin V.

\section{Abbreviations}

Ang II: Angiotensin II; Ang I: Angiotensin I; ARBs: Angiotensin receptor blockers; AMI: Acute myocardial infarction; LAD: The ligation of the left anterior descending coronary artery; VR: Ventricular remodeling; GXV: Guanxin V; Tra: Tranilast; LVIDd: Left ventricular end-diastolic inner diameter; LVIDs: Left ventricular end-systolic inner diameter; EF: Ejection fraction; FS: Fractional shortening; LVPWd: Left ventricular posterior wall end-diastolic thickness; LVPWs: Left ventricular posterior wall end-systolic thickness; LVEDV: Left ventricular end diastolic volume; LVESV: Left ventricular end systolic volume; HWI: Heart weight index; TB: Toluidine blue; H\&E: Hematoxylin and eosin; WB: Western blotting; PVDF: Polyvinylidene fluoride membranes; ELISA: Enzyme-linked immunosorbent assay; UPLC: Ultra-performance liquid chromatography; AT1R: Angiotensin II type1 receptor; MRAs: Mineralocorticoid receptor antagonists; SNS: Sympathetic nervous system; ECM: Extracellular matrix; MCs: Mast cells; RAAS: Reninangiotensin-aldosterone System; ACE: Angiotensin converting enzyme; ACEl: Angiotensin-converting enzyme inhibitor; AT2R: Angiotensin II type 2 receptor; TIC: Total ions chromatograph

\section{Acknowledgements}

We thank Juan Zhao, Xi-Zhong Yu, and Wen-Bin Shang, Medical Research Center of First College of Clinical Medicine, Nanjing University of Chinese Medicine, for their instructive advice and useful suggestions.

\section{Authors' contributions}

XX. Z, CL. S and SY. C performed animal experiments; XX. Z, Y. Z and B. L analyzed data; XX. Z drafted the manuscript; and N. G was responsible for experimental design. All authors have read and approved the manuscript.

\section{Funding}

This study was funded by the National Natural Science Foundation of China [NO.81774229], Jiangsu Leading Talent Project of Traditional Chinese Medicine [Jiangsu TCM 2018 No.4], Major Project of Nanjing Medical Science and Technology Development During 13th Five-year Plan [ZDX16013], and Jiangsu Universities Nursing Advantage Discipline Project [2019YSHL095].

\section{Availability of data and materials}

The datasets generated and/or analyzed during the current study are not publicly available since they are still under further study, but are available from the corresponding author on reasonable request.

Ethics approval and consent to participate

Animal procedures were approved by the Animal Ethics Committee, Nanjing University of Chinese Medicine (NO. 201809A005).

\section{Consent for publication}

Not applicable.

\section{Competing interests}

The authors declare that they have no competing interests.

\section{Author details}

${ }^{1}$ Nanjing University of Chinese Medicine, Nanjing, China. ${ }^{2}$ Department of Cardiology, Nanjing Hospital of Chinese Medicine, Affiliated to Nanjing University of Chinese Medicine, Daming Road 157\#, Nanjing 210000, Jiangsu, People's Republic of China. ${ }^{3}$ Xuzhou City Hospital of TCM, Xuzhou, China.

Received: 21 July 2020 Accepted: 10 January 2021

Published online: 22 February 2021

\section{References}

1. Thiele H, Ohman E. M, de Waha-Thiele S, Zeymer U and Desch S 2019 management of cardiogenic shock complicating myocardial infarction: an update. Eur Heart J. 2019;40:2671-83.

2. Tennant $\mathrm{R}$, Wiggers $\mathrm{CJ}$. The effect of coronary occlusion on myocardial contraction. Am J Physiol. 1935;112:351 AM HEART J 10 843-4.

3. Zhang BF, Jiang H, Chen J, Hu Q, Yang S, Liu XP, Liu G. LncRNA H19 ameliorates myocardial infarction-induced myocardial injury and maladaptive cardiac remodeling by regulating KDM3A. J Cell Mol Med. 2020:24:1099-115.

4. Bhatt AS, Ambrosy AP, Velazquez EJ. Adverse Remodeling and Reverse Remodeling After Myocardial Infarction. Curr Cardiol Rep. 2017;19:71.

5. Liang B, Zhang XX, Gu N. Virtual screening and network pharmacologybased synergistic mechanism identification of multiple components contained in Guanxin V against coronary artery disease. BMC Complement Med Ther. 2020;20(1):345.

6. Chang KS, Lee NH, Kuo WW, Hu WS, Chang MH, Tsai FJ, Tsai KH, Yang YS, Chen TS, Huang CY. Dung-Shen Downregulates the synergistic apoptotic effects of angiotensin II plus Leu 27-IGF II on Cardiomyoblasts. Acta Cardiol Sin. 2014;30:56-66.

7. Wang YB, Liu YF, et al. Rehmannia glutinosa extract activates endothelial progenitor cells in a rat model of myocardial infarction through a SDF-1 a CXCR4 cascade. PLoS One. 2013;8(1):54303.

8. Chen MH, Chen XJ, Wang M, Lin LG, Wang YT. Ophiopogon japonicus--a phytochemical, ethnomedicinal and pharmacological review. J Ethnopharmacol. 2016;181:193-213

9. Song $T$, Yao $Y$, Wang $T$, Huang $H$, Xia $H$. Tanshinone $\| A$ ameliorates apoptosis of myocardiocytes by up-regulation of miR-133 and suppression of Caspase-9. Eur J Pharmacol. 2017:815:343-50.

10. Ke Z, Wang G, Yang L, Qiu H, Wu H, Du M, Chen J, Song J, Jia X, Feng L. Crude terpene glycoside component from Radix paeoniae rubra protects against isoproterenol-induced myocardial ischemic injury via activation of the PI3K/AKT/mTOR signaling pathway. J Ethnopharmacol. 2017;206:160-9.

11. Zhang MY, Wu HW, Xu LP, Yang HJ. Pharmacological effect of Schisandrae Chinensis Fructus and relative active components on cardiovascular and cerebrovascular diseases. Zhongguo Zhong Yao Za Zhi. 2018;43:1536-46.

12. Komatsu H, Kojima M, Tsutsumi N, Hamano S, Kusama H, Ujiie A, Ikeda S, Nakazawa M. Study of the mechanism of inhibitory action of tranilast on chemical mediator release. Jpn J Pharmacol. 1988:46:43-51. 
13. Pae HO, Jeong SO, Koo BS, Ha HY, Lee KM, Chung HT. Tranilast, an orally active anti-allergic drug, up-regulates the anti-inflammatory heme oxygenase-1 expression but down-regulates the pro-inflammatory cyclooxygenase-2 and inducible nitric oxide synthase expression in RAW264.7 macrophages. Biochem Biophys Res Commun. 2008;371:361-5.

14. Kagitani S, Ueno H, Hirade S, Takahashi T, Takata M, Inoue H. Tranilast attenuates myocardial fibrosis in association with suppression of monocyte/ macrophage infiltration in DOCA/salt hypertensive rats. J Hypertens. 2004; 22:1007-15.

15. Shigeki S, Murakami T, Yata N, Ikuta Y. Treatment of keloid and hypertrophic scars by iontophoretic transdermal delivery of tranilast. Scand J Plast Reconstr Surg Hand Surg. 1997;31:151-8.

16. Nakatani Y, Nishida K, Sakabe M, Kataoka N, Sakamoto T, Yamaguchi Y, Iwamoto J, Mizumaki K, Fujiki A, Inoue H. Tranilast prevents atrial remodeling and development of atrial fibrillation in a canine model of atrial tachycardia and left ventricular dysfunction. J Am Coll Cardiol. 2013;61:582-8.

17. See F, Watanabe M, Kompa AR, Wang BH, Boyle AJ, Kelly DJ, Gilbert RE, Krum $\mathrm{H}$. Early and delayed tranilast treatment reduces pathological fibrosis following myocardial infarction. Heart Lung Circ. 2013;22:122-32.

18. Jun G, Wenbo J, et al. Clinical efficacy of Guanxin $\vee$ mixture in treating ventricular remodeling after acute myocardial infarction and its mechanism. China Pharmaceuticals. 2017;26(22):30-3.

19. Cao Y, He X, Lui F, Huang Z, Zhang Y. Chinese medicinal formula Guanxin Shutong capsule protects the heart against oxidative stress and apoptosis induced by ischemic myocardial injury in rats. Exp Ther Med. 2014;7:1033-9.

20. Ke F, Zuo KK, Ning G. Effects of Guanxin V preparation on rats ventricular remodeling and inflammatory factors with acute myocardial infarction. J Basic Chin Med. 2016;22(1):50-3.

21. Zhu J, Zhou H, Li C, He Y, Pan Y, Shou Q, Fang M, Wan H, Yang J. Guanxinshutong capsule ameliorates cardiac function and architecture following myocardial injury by modulating ventricular remodeling in rats. Biomed Pharmacother. 2020;130:110527.

22. Liang Z, Liu LF, Yao TM, Huo Y, Han YL. Cardioprotective effects of Guanxinshutong (GXST) against myocardial ischemia/ reperfusion injury in rats. J Geriatr Cardiol. 2012;9:130-6.

23. Liang B, Qu Y, Zhao QF, Gu N. Guanxin V for coronary artery disease: A retrospective study. Biomed Pharmacother. 2020;128:110280.

24. Cheng S, Zhang X, Feng Q, Chen J, Shen L, Peng Y, Yang L, Chen D, Zhang $H$, Sun W, Chen X. Astragaloside IV exerts angiogenesis and cardioprotection after myocardial infarction via regulating PTEN/PI3K/Akt signaling pathway[J]. Life Sci. 2019;227:82-93.

25. Reichert K, Colantuono B, McCormack I, Rodrigues F, Pavlov V, Ruhul Abid M. Murine left anterior descending (LAD) coronary artery ligation: an improved and simplified model for myocardial infarction[J]. Journal of visualized experiments : JoVE. 2017(122):55353.

26. Heidenreich PA, Albert NM, Allen LA, Bluemke DA, Butler J, Fonarow GC, Ikonomidis JS, Khavjou O, Konstam MA, Maddox TM, Nichol G, Pham M, Pina IL, Trogdon JG. Forecasting the impact of heart failure in the United States: a policy statement from the American Heart Association. Circ Heart Fail. 2013;6:606-19.

27. Pfeffer MA, Braunwald E. Ventricular remodeling after myocardial infarction. Experimental observations and clinical implications. Circulation. 1990;81:1161-72.

28. Cleutjens JP, Kandala JC, Guarda E, Guntaka RV, Weber KT. Regulation of collagen degradation in the rat myocardium after infarction. J Mol Cell Cardiol. 1995;27:1281-92

29. Warren SE, Royal HD, Markis JE, Grossman W, McKay RG. Time course of left ventricular dilation after myocardial infarction: influence of infarct-related artery and success of coronary thrombolysis. J Am Coll Cardiol. 1988;11:12-9.

30. Anversa P, Beghi C, Kikkawa Y, Olivetti G. Myocardial response to infarction in the rat. Morphometric measurement of infarct size and myocyte cellular hypertrophy. Am J Pathol. 1985;118:484-92.

31. Pfeffer JM, Pfeffer MA, Fletcher PJ, Braunwald E. Progressive ventricular remodeling in rat with myocardial infarction. Am J Physiol. 1991;260:H1406-14.

32. McKay RG, Pfeffer MA, Pasternak RC, Markis JE, Come PC, Nakao S, Alderman JD, Ferguson JJ, Safian RD, Grossman W. Left ventricular remodeling after myocardial infarction: a corollary to infarct expansion. Circulation. 1986;74: 693-702.

33. Benjamin MM, Smith RL, Grayburn PA. Ischemic and functional mitral regurgitation in heart failure: natural history and treatment. Curr Cardiol Rep. 2014;16:517.
34. Pfeffer MA, Braunwald E, Moye LA, Basta L, Brown EJ, Cuddy TE, Davis BR, Geltman EM, Goldman S, Flaker GC, Et A. Effect of captopril on mortality and morbidity in patients with left ventricular dysfunction after myocardial infarction. Results of the survival and ventricular enlargement trial. The SAVE Investigators. N Engl J Med. 1992;327:669-77.

35. Yousef ZR, Redwood SR, Marber MS. Postinfarction left ventricular remodelling: where are the theories and trials leading us? HEART. 2000;83:76-80.

36. Maczewski M, Borys M, Kacprzak P, Gdowski T, Kowalewski M, Wojciechowski D. Late ventricular remodeling in non-reperfused acute myocardial infarction in humans is predicted by angiotensin II type 1 receptor density on blood platelets. Int J Cardiol. 2008;127:57-63.

37. Hamon M, Filippi-Codaccioni E. The OPTIMMAL trial: losartan or captopril after acute myocardial infarction. Lancet. 2002;360:1886-7.

38. Doughty RN, Whalley GA, Walsh HA, Gamble GD, Lopez-Sendon J, Sharpe N. Effects of carvedilol on left ventricular remodeling after acute myocardial infarction: the CAPRICORN Echo Substudy. Circulation. 2004;109:201-6.

39. Kober L, Torp-Pedersen C, Carlsen JE, Bagger H, Eliasen P, Lyngborg K, Videbaek J, Cole DS, Auclert L, Pauly NC. A clinical trial of the angiotensinconverting-enzyme inhibitor trandolapril in patients with left ventricular dysfunction after myocardial infarction. Trandolapril Cardiac Evaluation (TRACE) Study Group. N Engl J Med. 1995;333:1670-6.

40. Roig E, Perez-Villa F, Morales M, Jimenez W, Orus J, Heras M, Sanz G. Clinical implications of increased plasma angiotensin II despite ACE inhibitor therapy in patients with congestive heart failure. Eur Heart J. 2000;21:53-7.

41. Chappell MC. Biochemical evaluation of the renin-angiotensin system: the good, bad, and absolute? Am J Physiol Heart Circ Physiol. 2016;310:H137-52.

42. Dahlin JS, Hallgren J. Mast cell progenitors: origin, development and migration to tissues. Mol Immunol. 2015;63:9-17.

43. Ahmad S, Simmons T, Varagic J, Moniwa N, Chappell MC, Ferrario CM. Chymase-dependent generation of angiotensin II from angiotensin-(1-12) in human atrial tissue. PLOS ONE. 2011;6:e28501.

44. Ahmad S, Ferrario C. M 2018 Chymase inhibitors for the treatment of cardiac diseases: a patent review. Expert Opin Ther Pat. 2010-2018;28:755-64.

\section{Publisher's Note}

Springer Nature remains neutral with regard to jurisdictional claims in published maps and institutional affiliations.

Ready to submit your research? Choose BMC and benefit from:

- fast, convenient online submission

- thorough peer review by experienced researchers in your field

- rapid publication on acceptance

- support for research data, including large and complex data types

- gold Open Access which fosters wider collaboration and increased citations

- maximum visibility for your research: over $100 \mathrm{M}$ website views per year

At BMC, research is always in progress.

Learn more biomedcentral.com/submissions 